ARTICLE

DOI: $10.1038 / s 41467-017-00309-w$

\title{
Micromotor-enabled active drug delivery for in vivo treatment of stomach infection
}

Berta Esteban-Fernández de Ávila1, Pavimol Angsantikul (10 1, Jinxing Li ${ }^{1}$, Miguel Angel Lopez-Ramirez ${ }^{1}$, Doris E. Ramírez-Herrera1, Soracha Thamphiwatana1, Chuanrui Chen', Jorge Delezuk1, Richard Samakapiruk1, Valentin Ramez ${ }^{1}$, Marygorret Obonyo ${ }^{2}$, Liangfang Zhang (iD ${ }^{1} \&$ Joseph Wang ${ }^{1}$

Advances in bioinspired design principles and nanomaterials have led to tremendous progress in autonomously moving synthetic nano/micromotors with diverse functionalities in different environments. However, a significant gap remains in moving nano/micromotors from test tubes to living organisms for treating diseases with high efficacy. Here we present the first, to our knowledge, in vivo therapeutic micromotors application for active drug delivery to treat gastric bacterial infection in a mouse model using clarithromycin as a model antibiotic and Helicobacter pylori infection as a model disease. The propulsion of drug-loaded magnesium micromotors in gastric media enables effective antibiotic delivery, leading to significant bacteria burden reduction in the mouse stomach compared with passive drug carriers, with no apparent toxicity. Moreover, while the drug-loaded micromotors reach similar therapeutic efficacy as the positive control of free drug plus proton pump inhibitor, the micromotors can function without proton pump inhibitors because of their built-in proton depletion function associated with their locomotion.

\footnotetext{
${ }^{1}$ Department of NanoEngineering, University of California San Diego, La Jolla, CA 92093, USA. ${ }^{2}$ Department of Medicine, University of California San Diego, La Jolla, CA 92093, USA. Berta Esteban-Fernández de Ávila, Pavimol Angsantikul and Jinxing Li contributed equally to this work. Correspondence and requests for materials should be addressed to L.Z. (email: zhang@ucsd.edu) or to J.W. (email: josephwang@ucsd.edu)
} 
R ecent advances in the nano and micromotor field ${ }^{1-4}$ in terms of improvement of biocompatibility and biological function have led to their growing use in biomedicine ${ }^{5-7}$, including therapeutic payload delivery ${ }^{8-13}$, micro-surgery ${ }^{14}, 15$, isolation of biological targets ${ }^{16}$, operation within living cells ${ }^{17,18}$, and removal of toxicant molecules and organisms ${ }^{19-2}$. Although significant progress has been accomplished to demonstrate the in vitro capabilities of nano/micromotors to transport therapeutic cargos to target destinations, tremendous effort is still required to translate the proof-of-concept research to in vivo biomedical applications.

In recent years, the utility and performance of these motor-based active transport systems have been tested in live animals. For example, our group has demonstrated the attractive in vivo performance of zinc-based and magnesium $(\mathrm{Mg})$-based micromotors under in vivo conditions ${ }^{22-24}$. These studies have shown that artificial micromotors can self-propel in the stomach, and intestinal fluids for enhanced retention in the gastric mucous layer ${ }^{22}$ and targeted delivery in the gastrointestinal (GI) $\operatorname{tract}^{23}$.
Walker et al. ${ }^{25}$ presented the ability of magnetic micropropellers to move through gastric mucin gels, by mimicking the mucus penetration strategy of Helicobacter pylori (H. pylori). In addition, Nelson's group has demonstrated that magnetically actuated microswimmers can swarm in vivo ${ }^{11}$, whereas Martel's group has shown that microorganisms can be transformed into natural robots under magnetic guidance towards therapeutic cargo delivery into deep tumor regions ${ }^{12}$. These prior in vivo studies of synthetic motors have significantly advanced motor research and cleared a path towards direct evaluation of disease-oriented therapeutic efficacy associated with motor-enabled active drug delivery. However, this still remains an alluring but unmet goal for biomedical researchers.

This work demonstrates, to the best of our knowledge, the first attempt to apply Mg-based micromotors, loaded with antibiotic drug clarithromycin (CLR), for in vivo treatment of $H$. pylori infection in a mouse model. Given the built-in proton depletion function, this motor-based therapy is able to undergo the harsh gastric environment to achieve antibacterial efficacy a

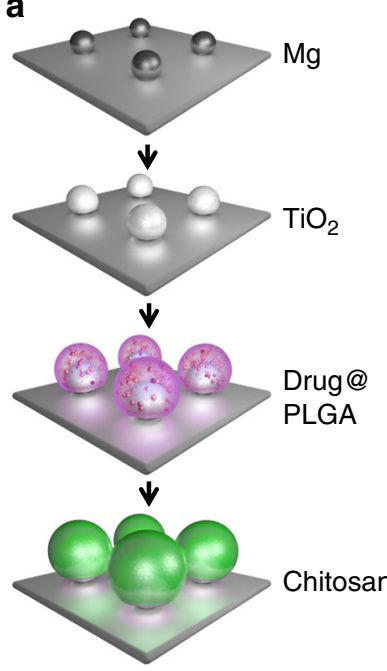

b

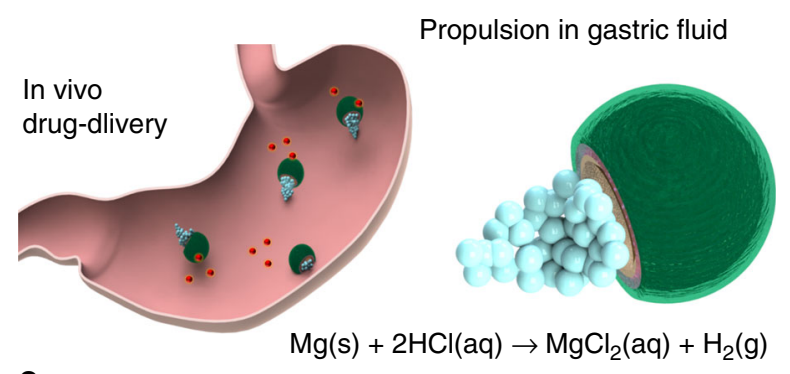

C
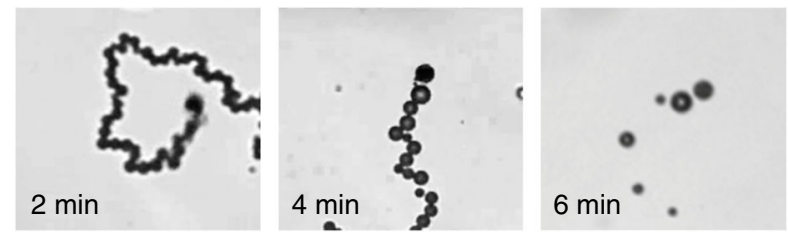

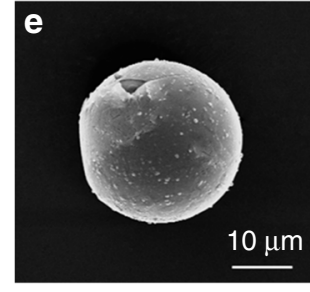

i

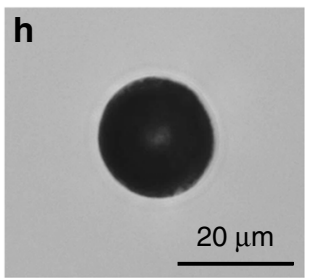

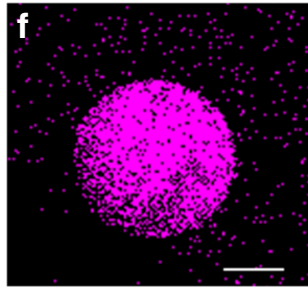
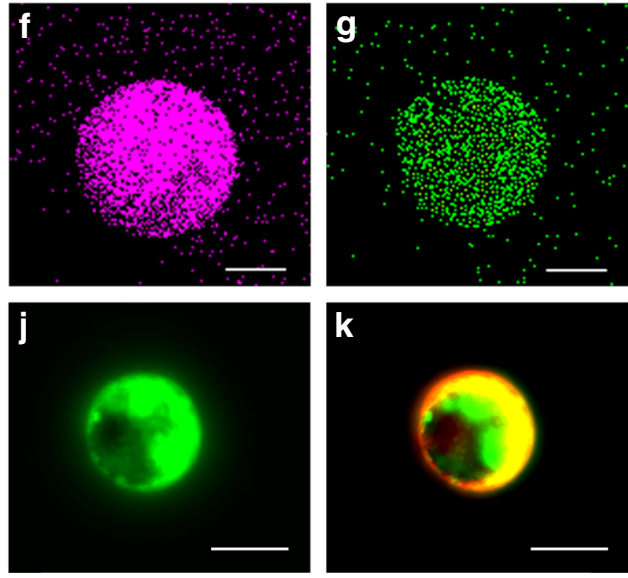

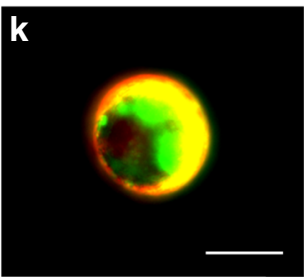

Fig. 1 Synthesis and characterization of drug-loaded Mg-based micromotors. a Schematic preparation of the micromotors: Mg microparticles dispersion over a glass slide, $\mathrm{TiO}_{2}$ atomic layer deposition (ALD) over the $\mathrm{Mg}$ microparticles, drug-loaded PLGA deposition over the $\mathrm{Mg}^{-\mathrm{TiO}} \mathrm{m}_{2} \mathrm{microparticles}$, and Chitosan polymer deposition over the $\mathrm{Mg}-\mathrm{TiO}_{2}-\mathrm{PLGA}$ microparticles. b Schematic of in vivo propulsion and drug delivery of the $\mathrm{Mg}$-based micromotors in a mouse stomach. c Time-lapse images ( 2 min intervals, taken from Supplementary Movie 3 ) of the propulsion of the drug-loaded Mg-based micromotors in simulated gastric fluid ( $\mathrm{pH}$ 1.3). d Schematic dissection of a drug-loaded micromotor consisting of a Mg core, a TiO ${ }_{2}$ shell coating, a drug-loaded PLGA layer, and a chitosan layer. e Scanning electron microscopy (SEM) image of a drug-loaded Mg-based micromotor. $\mathbf{f}, \mathbf{g}$ Energy-dispersive X-ray spectroscopy (EDX) images illustrating the distribution of $\mathbf{f}$ magnesium and $\mathbf{g}$ titanium in the micromotor. h-k Microscopy images of dye-loaded Mg-based micromotor: $\mathbf{h}$ optical image and fluorescence images showing the dye-loaded Mg-based micromotors in the $\mathbf{i}$ DiD channel (PLGA layer), j FITC channel (chitosan layer), along with an overlay of the two channels $\mathbf{k}$ 
without involving the commonly used proton pump inhibitors (PPIs). The H. pylori bacteria, found in about half of the world's population, can cause stomach infection and subsequently lead to diverse gastric and extragastric diseases ${ }^{26,27}$. In most cases, the administration of antibiotics for the treatment of $\mathrm{H}$. pylori infection is combined with the use of PPIs to reduce the production of gastric acid ${ }^{28}$, because the gastric acid could make antibiotics less effective. The effectiveness of PPIs is attributed to the irreversible binding to proton pumps and thus to suppress acid secretion ${ }^{29}, 30$, which in long term use can lead to adverse effects such as headache and diarrhea and in more serious scenarios cause anxiety or depression ${ }^{31-34}$. Therefore, it would be highly beneficial to develop an alternative therapeutic regimen with equivalent or advantageous therapeutic efficacy as the current antibiotic treatments while excluding the use of PPIs.

The reported Mg-based micromotors rely on the combination of a CLR-loaded poly(lactic-co-glycolic acid) (PLGA) layer and a chitosan polymer layer covering on a propellant $\mathrm{Mg}$ core to offer high drug-loading capacity, along with biodegradability. The positively charged chitosan outer coating enables adhesion of the motor onto the stomach wall ${ }^{35}$, facilitating efficient localized autonomous release of CLR from the PLGA polymer coating. In contrast to acid suppression by PPIs, Mg-based micromotors can temporally and physically alter the local acidic environment by quickly depleting protons while propelling within the stomach $^{24}$. By using acid as fuel, these synthetic motors rapidly deplete protons while propelling within the stomach, which can effectively elevate the gastric $\mathrm{pH}$ to neutral in $<20 \mathrm{~min}$ after the motors are applied ${ }^{24}$. Testing in a mouse model has demonstrated that these motors can safely and rapidly neutralize gastric acid without causing noticeable acute toxicity or affecting the stomach function, and that the normal stomach $\mathrm{pH}$ can be restored within $24 \mathrm{~h}$ post motor administration. Such elimination of the PPI administration is coupled with significant reduction of bacteria burden, as demonstrated in vivo in a mouse model. Using a mouse model of $H$. pylori infection, the propulsion of the drug-loaded $\mathrm{Mg}$-based micromotors in gastric fluid along with their outer chitosan layer are shown to greatly enhance the binding and retention of the drug-loaded motors on the stomach wall. As these micromotors are propelled in the gastric fluid, their $\mathrm{Mg}$ cores are dissolved, leading to self-destruction of these motors without harmful residues, as is demonstrated by the toxicity studies.

Overall, we take advantage of the efficient propulsion of Mg-based micromotors in the acidic stomach environment, their built-in proton depletion ability, their active and prolonged retention within the stomach wall, and their high drug-loading capacity, to demonstrate to the best of our knowledge the first actual in vivo therapeutic application of chemically powered micromotors. In vivo studies examine the therapeutic efficacy, distribution, and retention of the micromotors in the mouse stomach compared with passive drug-loaded microparticles and other control groups, along with the corresponding in vivo toxicity profile. These results illustrate the attractive therapeutic capabilities of acid-driven micromotors, which open the door for in vivo therapeutic applications of body-fluid propelled micromotors towards the treatment of a variety of diseases and disorders.

\section{Results}

Drug-loaded Mg-micromotors preparation and characterization. Figure $1 \mathrm{a}$ and Supplementary Movie 1 schematically illustrate the preparation steps of the drug-loaded Mg-based micromotors. The cores of the micromotors are made of $\mathrm{Mg}$ microparticles with an average size of $\sim 20 \mu \mathrm{m}$. In the study, a layer of $\mathrm{Mg}$ microparticles was dispersed onto a glass slide, followed by an asymmetrical coating of the microspheres with a thin $\mathrm{TiO}_{2}$ layer using atomic layer deposition (ALD). The ALD process leads to a $\mathrm{TiO}_{2}$ uniform coating over the Mg-microspheres, while leaving a small opening (essential for contact with the acid fuel) at the sphere-glass contact point ${ }^{36}$, which forms a Janus microstructure. Such $\mathrm{TiO}_{2}$ layer acts as a shell scaffold that maintains the micromotor spherical shape and the opening size during the propulsion, leading to consistent and prolonged operation. The $\mathrm{Mg}-\mathrm{TiO}_{2}$ Janus microparticles were then coated with a PLGA film containing the CLR antibiotic payload. After the drug-loading step, the microparticles were coated with an outer thin chitosan layer (thickness $~ 100 \mathrm{~nm}$ ) that ensures efficient electrostatic adhesion of the micromotors to the mucosal layer on the stomach wall while protecting the CLR-loaded PLGA layer. Finally, the resulting CLR-loaded Mg-based micromotors were separated and collected by soft mechanical scratching of the glass slide, leaving a small opening for spontaneous Mg-acid reaction when the motors are placed in an acidic solution. This reaction generates hydrogen microbubbles and leads to efficient propulsion in the stomach fluid ${ }^{24}$. The small opening enables also a slow reaction process and gradual dissolution of the $\mathrm{Mg}$ core, leading to a prolonged micromotor lifetime of $\sim 6 \mathrm{~min}$. The in vivo self-propulsion in the gastric fluid of a stomach and the corresponding drug delivery process from the PLGA layer of the Mg-based micromotors are illustrated schematically in Fig. $1 \mathrm{~b}$ and Supplementary Movie 2.

The ability of drug-loaded Mg-based micromotors to efficiently propel in gastric acid was first tested in vitro by using a simulated gastric fluid ( $\mathrm{pH} \sim 1.3$ ). The microscopic images in Fig. 1c (taken from Supplementary Movie 3 at 2 min intervals) illustrate the fast and prolonged autonomous propulsion of a CLR-loaded Mg-based micromotor in the gastric fluid simulant. The efficient hydrogen bubble generation propels the micromotors rapidly, with an average speed of $\sim 120 \mu \mathrm{m} \mathrm{s}^{-1}$ (corresponding to a relative speed of 6 body length $\mathrm{s}^{-1}$ ), and indicates that the Mg-based micromotors can react and move fast in the gastric fluid. Such efficient micromotor propulsion is essential for the motors to reach stomach wall and thus achieving significant therapeutic efficacy. Importantly, the acid-Mg reaction responsible for the autonomous propulsion also spontaneously depletes protons in gastric fluid and thus neutralizes the stomach $\mathrm{pH}$ without using $\mathrm{PPIs}^{24}$.

Figure 1d schematically illustrates the structure of a drug-loaded Mg-based micromotor, showing the $\mathrm{Mg}$ core, covered mostly with the $\mathrm{TiO}_{2}$ shell layer, drug-loaded PLGA layer, and an outer chitosan layer. The drug-loaded Mg-based micromotors were carefully characterized. The scanning electron microscopy (SEM) image of a drug-loaded micromotor (shown in Fig. 1e) confirms the presence of a small opening $(\sim 2 \mu \mathrm{m})$ on the spherical micromotor, produced during the coating process, that exposes the $\mathrm{Mg}$ core of the micromotor to the gastric fluid and facilitates the hydrogen bubble thrust. Energy-dispersive X-ray (EDX) spectroscopy mapping analysis was carried out to confirm the motor composition. The resulting EDX images, shown in Fig. If and g, illustrate the presence and distribution of magnesium and titanium, respectively.

A fluorescence study was carried out to confirm efficient drug-loading within the PLGA layer, and the coating of the micromotor with the protective and adhesive chitosan layer. This was accomplished by preparing Mg-based micromotors with the PLGA and chitosan coatings containing the fluorescent dyes 1,1'-dioctadecyl-3,3,3',3'-tetramethylindodicarbocyanine, 4-chlorobenzenesulfonate salt $\left(\mathrm{DiD}, \quad \lambda_{\mathrm{em}}=665 \mathrm{~nm}\right), \quad$ and fluorescein isothiocyanate-dextran (FITC, $\lambda_{\mathrm{em}}=520 \mathrm{~nm}$ ), 
a
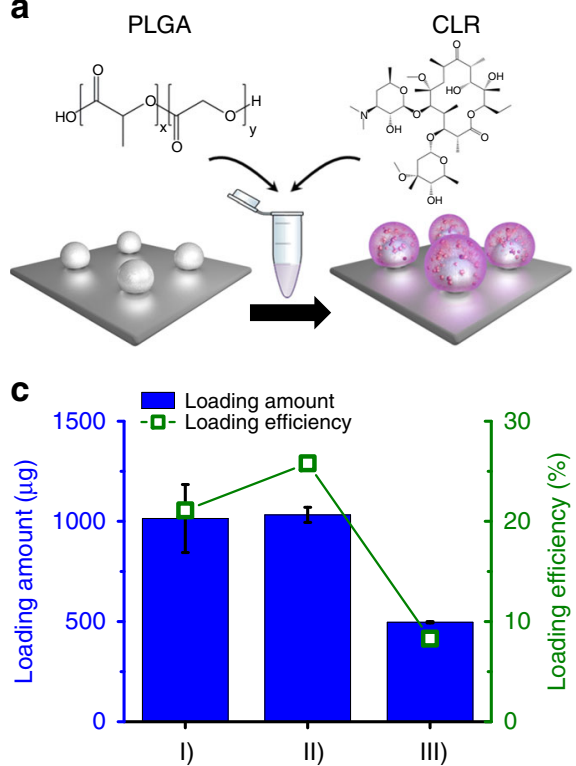

b

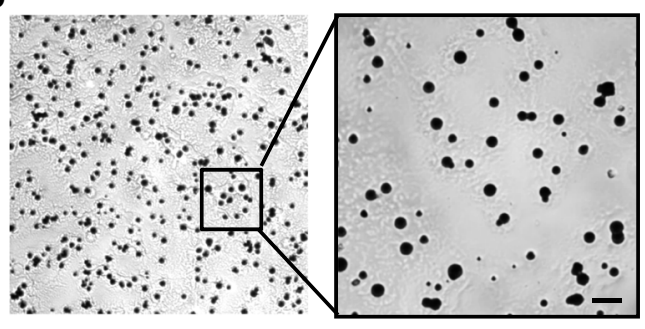

d

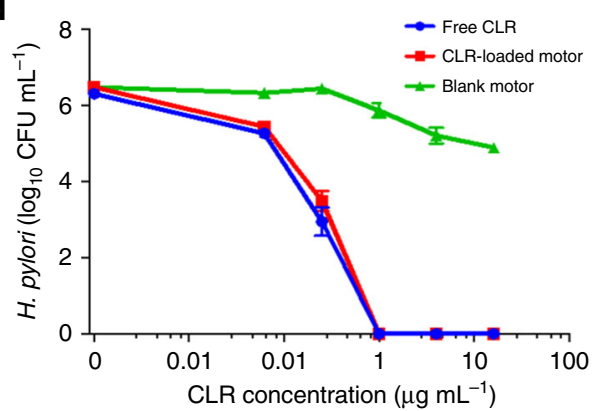

Fig. 2 Antibiotic drug loading of the Mg-based micromotors and in vitro bactericidal activity. a Schematic displaying the loading clarithromycin (CLR) onto the $\mathrm{Mg}$-based micromotors. PLGA polymer dissolved in ethyl acetate is mixed with $\mathrm{CLR}$, and the solution is deposited over the $\mathrm{Mg}^{-\mathrm{TiO}} \mathrm{O}_{2}$ microparticles resulting in the formation of a thin PLGA-CLR coating. b Microscope images showing the PLGA-CLR film over the Mg-based micromotors. Scale bars 100 $\mu \mathrm{m}$ and $40 \mu \mathrm{m}$, respectively. c Quantification of CLR-loading amount and yield of the micromotors prepared with different CLR solutions: (I) $100 \mu \mathrm{L}$ of 40 $\mathrm{mg} \mathrm{mL}^{-1} \mathrm{CLR}$ solution, (II) $120 \mu \mathrm{L}$ of $40 \mathrm{mg} \mathrm{mL}^{-1} \mathrm{CLR}$ solution, and (III) $200 \mu \mathrm{L}$ of $30 \mathrm{mg} \mathrm{mL}^{-1} \mathrm{CLR}$ solution. All the CLR-loaded Mg-based micromotors were coated with a thin chitosan layer; all samples were dissolved in acid for $24 \mathrm{~h}$ before the drug-loading measurement. $\mathbf{d}$ In vitro bactericidal activity of free CLR, CLR-loaded Mg-based micromotors, and blank Mg-based micromotors (without CLR drug) against $H$. pylori bacteria. Error bars estimated as a triple of s.d. $(n=3)$

respectively. An optical image of a dye-loaded micromotor is displayed in Fig. 1h. The corresponding fluorescence images show the dye-loaded Mg-based micromotor in the DiD and FITC channels (Fig. 1i and j, respectively); an overlay of the two channels is displayed in Fig. 1k. The high-fluorescent intensity of the loaded dyes confirms the successful coating of the micromotor with both PLGA and chitosan layers, along with the high cargo-loading capacity of the micromotor.

Prior to in vivo therapeutic application of the Mg-based micromotors, several in vitro studies were performed. Initially, the ability of drug-loaded micromotors to efficiently propel in gastric acid was tested in vitro. Supplementary Fig. 1a-d displays time-lapse images (corresponding to Supplementary Movie 4) showing the motion of the drug-loaded Mg-based micromotors in simulated gastric fluid adjusted to different $\mathrm{pH}$ values $(0.75,1.25$, 1.5 , and 1.75 , respectively). Time-lapse images in Supplementary Fig. 1e-h show the lifetime of a drug-loaded micromotor in gastric fluid simulant ( $\mathrm{pH} \sim 1.3$ ) to be $\sim 6 \mathrm{~min}$. Supplementary Fig. 1i displays the $\mathrm{pH}$-dependent speed of the micromotor in the gastric fluid simulant. The micromotor speed drastically decreases upon changing the $\mathrm{pH}$ of the gastric fluid solution from $\mathrm{pH}$ $1.5-1.75$. Assuming that the stomach $\mathrm{pH}$ is 1.3 , the drug-loaded Mg-based micromotors can efficiently move at this condition with an average speed of $\sim 120 \mu \mathrm{m} \mathrm{s}^{-1}\left(\sim 6\right.$ body length $\left.\mathrm{s}^{-1}\right)$.

Drug-loading optimization and in vitro bactericidal activity. The CLR-loading onto the Mg-based micromotors was optimized to achieve a clinically relevant therapeutic concentration of the drug (15-30 mg kg $\left.{ }^{-1} \mathrm{day}^{-1}\right)^{37}$. Figure 2a shows a schematic displaying the loading of CLR onto the micromotors. Briefly, the $\mathrm{Mg}-\mathrm{TiO}_{2}$ microparticles dispersed onto a glass slide ( $2 \mathrm{mg}$ of $\mathrm{Mg}$ microparticles per glass slide) were coated with a PLGA solution prepared in ethyl acetate, which was mixed with CLR (see detailed experimental protocol in "Methods" section). Rapid evaporation under nitrogen current leads to the formation of a homogeneous PLGA-CLR coating over the $\mathrm{Mg}-\mathrm{TiO}_{2}$ microparticles (microscope images of the coated micromotors are displayed in Fig. 2b). The microparticles were further coated with chitosan before quantifying the CLR-loading efficiency of the micromotors. To optimize the drug-loading, Mg-based micromotors were coated with PLGA solutions containing different amounts of CLR (between 4 and $6 \mathrm{mg}$ ). By studying different combinations of the PLGA-CLR solution volume and CLR concentration, the highest CLR-loading efficiency (26\%), corresponding to $1032 \pm 37 \mu \mathrm{g}$ per $2 \mathrm{mg}$ micromotor, was obtained when coating the microparticles with $120 \mu \mathrm{L}$ of the PLGA solution containing $4.8 \mathrm{mg}$ of CLR (Fig. 2c, II). This formulation offered optimal CLR-loading and was selected for subsequent in vitro and in vivo anti- $H$. pylori studies.

Once confirmed that the micromotors were capable to load antibiotic cargo with high-loading efficiency, an in vitro bactericidal activity of CLR-loaded Mg-based micromotors against $H$. pylori was performed. To mimic the gastric environment, samples were treated in $0.1 \mathrm{~N} \mathrm{HCl}$ for $1 \mathrm{~h}$ prior to incubation with bacteria. This also ensured the dissolution of micromotors and consecutive drug release. Figure $2 \mathrm{~d}$ shows the enumerated amount of bacteria after being treated by CLR-loaded Mg-based micromotors or free CLR solution with varying concentrations of CLR. According to the results, drug-loaded micromotors exhibited a comparable bactericidal activity to free drug solution over the whole range of concentrations used in the study. Specifically, we determined the minimal bactericidal concentration (MBC) values of the samples, defined as the minimal concentration of an antimicrobial agent that kills 3 logs (99.9\%) of the bacteria. The MBC value for CLR-loaded Mg-based micromotors was found to be $0.25 \mu \mathrm{g} \mathrm{mL}^{-1}$, which was unaltered from the MBC value of free CLR. Moreover, bare 
a
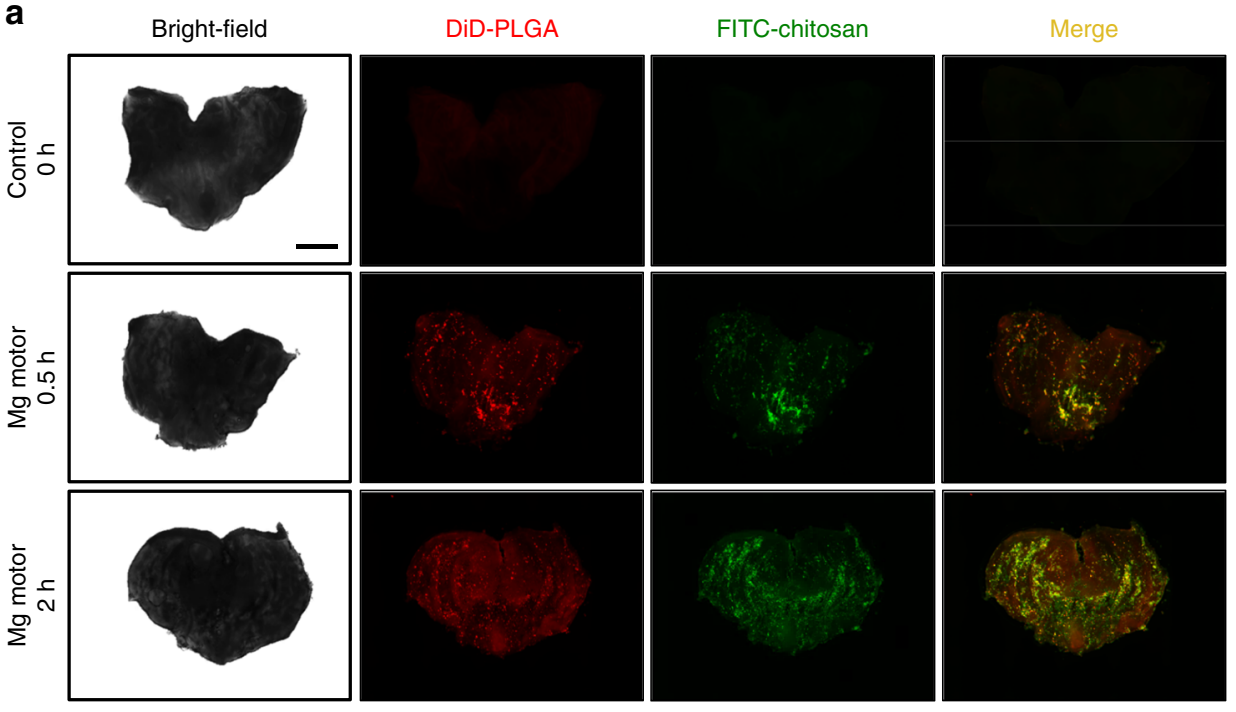

b

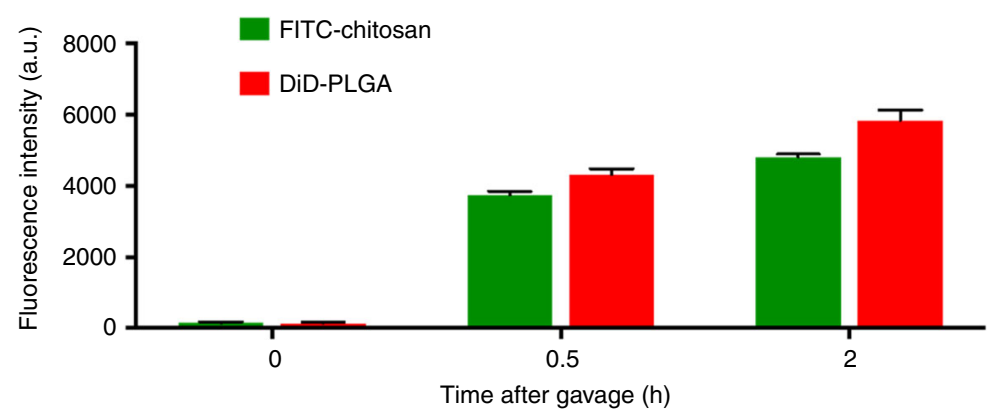

Fig. 3 Retention of the Mg-based micromotors in mouse stomachs. a Bright-field and fluorescence images of the luminal lining of freshly excised mouse stomachs at $0 \mathrm{~min}$ after oral gavage of deionized (DI) water (control), and at $30 \mathrm{~min}$ and $2 \mathrm{~h}$ after oral gavage of the $\mathrm{Mg}$-based micromotors. Scale bar 500 $\mathrm{mm}$. b Corresponding fluorescence quantification of all the images shown in a. Error bars estimated as a triple of s.d. $(n=3)$

Mg-based micromotors, with corresponding amount of motors and treated under the same conditions as the free CLR and CLR-loaded Mg-micromotors, were used as negative controls. From Fig. 2d, the bare motors had negligible effect on the viability of $H$. pylori over the studied range, which supports that the bactericidal effect of CLR-loaded Mg-based micromotors is solely due to the loaded antibiotics, and not due the other compositions of the micromotor carrier or the micromotor acidic environment. Overall, Fig. 2d verifies that the activity of the loaded drug was not compromised compared to free drug. Our in vitro results verified also that drug-loaded micromotors, made of $\mathrm{Mg}$ and other degradable materials, eventually destroy themselves and disappear in the acidic environment after releasing the CLR, with no apparent residues in the tissue. The findings validate the potential use of these drug-loaded micromotors for therapeutic applications.

In vivo micromotor retention in mouse stomach. After the optimization of drug-loading onto the Mg-based micromotors and the confirmation of effective in vitro bactericidal activity, the micromotors were further investigated under in vivo setting. First, the in vivo retention properties of the Mg-based micromotors on stomach tissue were examined at different post-administration times, and compared with control groups administered with DI water (Fig. 3). For this purpose, Mg-based micromotors prepared with DiD-labeled PLGA and FITC-labeled chitosan coatings were administered to a group of mice $(n=3)$, and following $30 \mathrm{~min}$ and $2 \mathrm{~h}$ of the samples administration, the mice were killed and the entire stomach was excised and opened.
Subsequently, the luminal lining was rinsed with PBS and flattened for imaging. Accordingly, Fig. 3a shows bright-field and fluorescence images of the luminal lining of freshly excised mouse stomach at $0 \mathrm{~min}$ after oral gavage of DI water, and at $30 \mathrm{~min}$ and $2 \mathrm{~h}$ after oral gavage of Mg-based micromotors. As can be observed, the images corresponding to the dye-loaded Mg-based micromotors show an intense fluorescent signal in both red and green light channels, which indicates efficient distribution and retention of the micromotors in the mouse stomach. The continuous propulsion of the micromotors and the adhesive properties of the chitosan coating help to achieve a homogeneous distribution of the micromotors in the stomach. The corresponding fluorescence quantification of the dye-loaded micromotors retained in the mouse stomach after $30 \mathrm{~min}$ and $2 \mathrm{~h}$ oral gavage of the sample is displayed in Fig. $3 \mathrm{~b}$. The graphic represents the higher fluorescence signals obtained at 665 and $520 \mathrm{~nm}$ (corresponding to DiD and FITC dyes, respectively) for each sample. These results indicate that the micromotors can effectively propel in gastric fluid and are retained in the stomach wall, including the antrum, where the $H$. pylori bacteria reside. Such highly enhanced retention in the stomach, which is a major advantage of motor-enabled delivery, has been carefully examined in our early studies ${ }^{22-24}$. The powerful propulsion leads to tissue penetration and binding, so that the drug-loaded motor could reach the whole stomach wall for enhanced retention.

In vivo anti-H. pylori therapeutic efficacy. We proceeded to test the in vivo therapeutic efficacy of the drug-loaded Mg-based micromotors against $H$. pylori infection. Prior to the therapeutic 

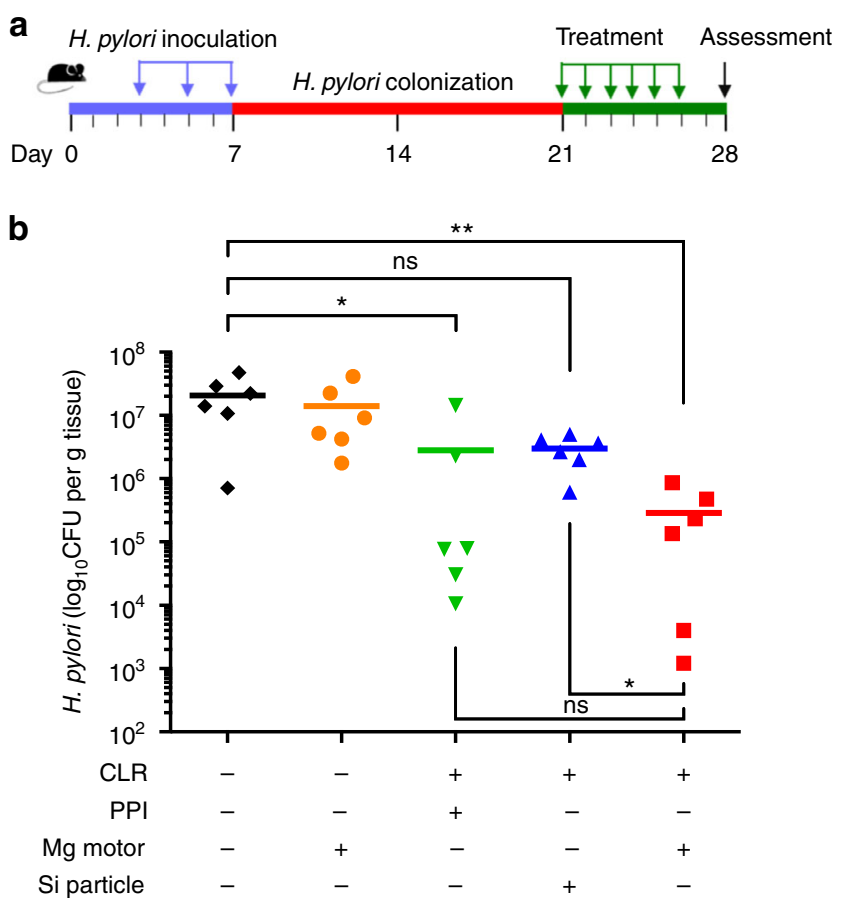

Fig. 4 In vivo anti-H. pylori therapeutic efficacy. a The study protocol including H. pylori inoculation and infection development in C57BL/6 mice, followed by the treatments. b Quantification of bacterial burden in the stomach of $\mathrm{H}$. pylori-infected mice treated with DI water (black color), bare Mg-based micromotors (orange color), free CLR+PPI (green color), CLRloaded silica microparticles (blue color), and CLR-loaded Mg-based micromotors (red color), respectively ( $n=6$ per group). Bars represent median values. ${ }^{\star} P<0.05,{ }^{\star}{ }^{\star} P<0.01$, ns no statistical significance

study, we developed $H$. pylori infection in a mouse model using C57BL/6 mice. Each mouse was inoculated with $3 \times 10^{8} \mathrm{CFU}$ H. pylori SS1 in brain-heart infusion (BHI) broth by oral gavage three times on day 3, 5, and 7 (Fig. 4a) ${ }^{38,39}$. Two weeks after inoculation, the $H$. pylori-infected mice were divided into five groups ( $n=6$, for each group) and orally administered with DI water, blank Mg-based micromotors (without CLR drug), free CLR drug with PPI (CLR+PPI), CLR-loaded silica microparticles, or CLR-loaded Mg-based micromotors once a day for five consecutive days. On each day of treatment, mice in the free CLR+PPI group received $400 \mu \mathrm{mol} \mathrm{kg}-1$ of omeprazole (as PPI treatment) $30 \mathrm{~min}$ before administrating CLR, to neutralize gastric acid and prevent potential degradation of CLR. Such PPI dosage has been reported to be effective both in reducing the gastric acidity in mouse models ${ }^{40}$, as well as in preserving the effectiveness of co-administered antibiotics $39,41,42$. After the treatment course, the bacterial burden was evaluated by enumerating and comparing $H$. pylori counts recovered from each mouse stomach. The mean bacterial burden from two negative control groups treated with DI water and blank Mg-based motors were $2.1 \times 10^{7}$ and $1.4 \times 10^{7} \mathrm{CFU} \mathrm{g}^{-1}$ of stomach tissue, respectively (Fig. 4b, black and orange color, respectively). Meanwhile, a bacterial burden of $3 \times 10^{6} \mathrm{CFU} \mathrm{g}^{-1}$ was measured from the mice treated with CLR-loaded silica microparticles, which did not show statistical difference to the negative controls. In contrast, when the mice were treated with CLR-loaded Mg-based micromotors, the bacterial burden was quantified as $2.9 \times 10^{5} \mathrm{CFU} \mathrm{g}^{-1}$, a significant reduction compared with the negative control and CLR-loaded silica microparticle groups. The substantial improvement in $H$. pylori reduction demonstrates the benefit of acid-powered $\mathrm{Mg}$-based micromotors compared with static micron-sized carriers. A bacterial burden of $2.8 \times 10^{6} \mathrm{CFU} \mathrm{g}^{-1}$ was obtained for the positive control mice with free CLR+PPI treatment. Although the difference between CLR-loaded Mg-based micromotors and the free CLR+PPI groups was not statistically significant, the CLR-loaded micromotors reduced the $H$. pylori burden in mice compared with in the negative controls by $\sim 1.8$ orders of magnitude, whereas the free CLR+PPI group reduced it only by $\sim 0.8$ orders of magnitude. These results might be derived from the benefit of the propulsion-enabled active drug delivery performed by the Mg-based micromotors in the stomach. These results demonstrate that the Mg-based micromotors can effectively propel and distribute throughout the stomach of living mice to significantly reduce $H$. pylori levels.

In vivo toxicity evaluation of Mg-based micromotors. Finally, the toxicity profile of the Mg-based micromotors in the stomach as well as in the lower GI tract was evaluated. Healthy mice were orally administered with Mg-based micromotors or DI water once daily for five consecutive days. Throughout the treatment, no signs of distress such as squinting of eyes, hunched posture, unkempt fur, or lethargy were observed in both groups. Initially, the toxicity profile of the Mg-micromotors in the mouse was evaluated through changes in body weight. During the experimental period, mice administered Mg-micromotors maintained a constant body weight compared with the mice administered DI water (Fig. 5a). On day 6, mice were killed and their stomachs and lower GI sections were processed for histological staining. Longitudinal sections of the glandular stomach (Fig. 5b), three major segments of small intestine (duodenum, jejunum, and ileum, Fig. 5c-e, respectively) and the two major segments of large intestine (proximal and distal colon, Fig. 5f-g, respectively) were stained with hematoxylin and eosin (H\&E). The stomach and lower GI sections of the micromotor-treated group showed undamaged structure of columnar epithelial cells with no signs of superficial degeneration or erosion (Fig. 5b-g, left). There was no noticeable difference in the gastric and intestinal mucosal integrity, in terms of thickness as well as size and number of crypt and villus, between the motor-treated and DI water-treated groups (Fig. 5b-g, left vs. right part). No lymphocytic infiltration into the mucosa and submucosa was observed, indicating no sign of gastric inflammation. The in vivo toxicity studies of $\mathrm{Mg}$-based micromotors showed no effect on the mouse body weight, apparent alteration of GI histopathology or observable inflammation, suggesting that the treatment of Mg-based micromotors is safe in the mouse model.

\section{Conclusions}

In this work we conducted the first, to the best of our knowledge, study to evaluate the therapeutic efficacy of a drug-loaded $\mathrm{Mg}$-based micromotor for in vivo treatment of $H$. pylori infection in a mouse model. Through these in vivo experiments, we demonstrated that acid-powered Mg-based micromotors could efficiently be loaded with clinical doses of drugs, retain in the mouse stomach wall, and perform an appreciable in vivo bactericidal activity. Our results showed that the active propulsion of drug-loaded $\mathrm{Mg}$-based micromotors in the acidic media of the stomach and motor-tissue interaction lead to efficient drug delivery and hence to a significant reduction of bacteria burden compared to passive drug carriers. Furthermore, such drug-loaded micromotors function in gastric condition for the H.pylori infection treatment without the need of PPIs. We also demonstrated that there were no toxicological consequences of the micromotors in the mouse models. Overall, our 

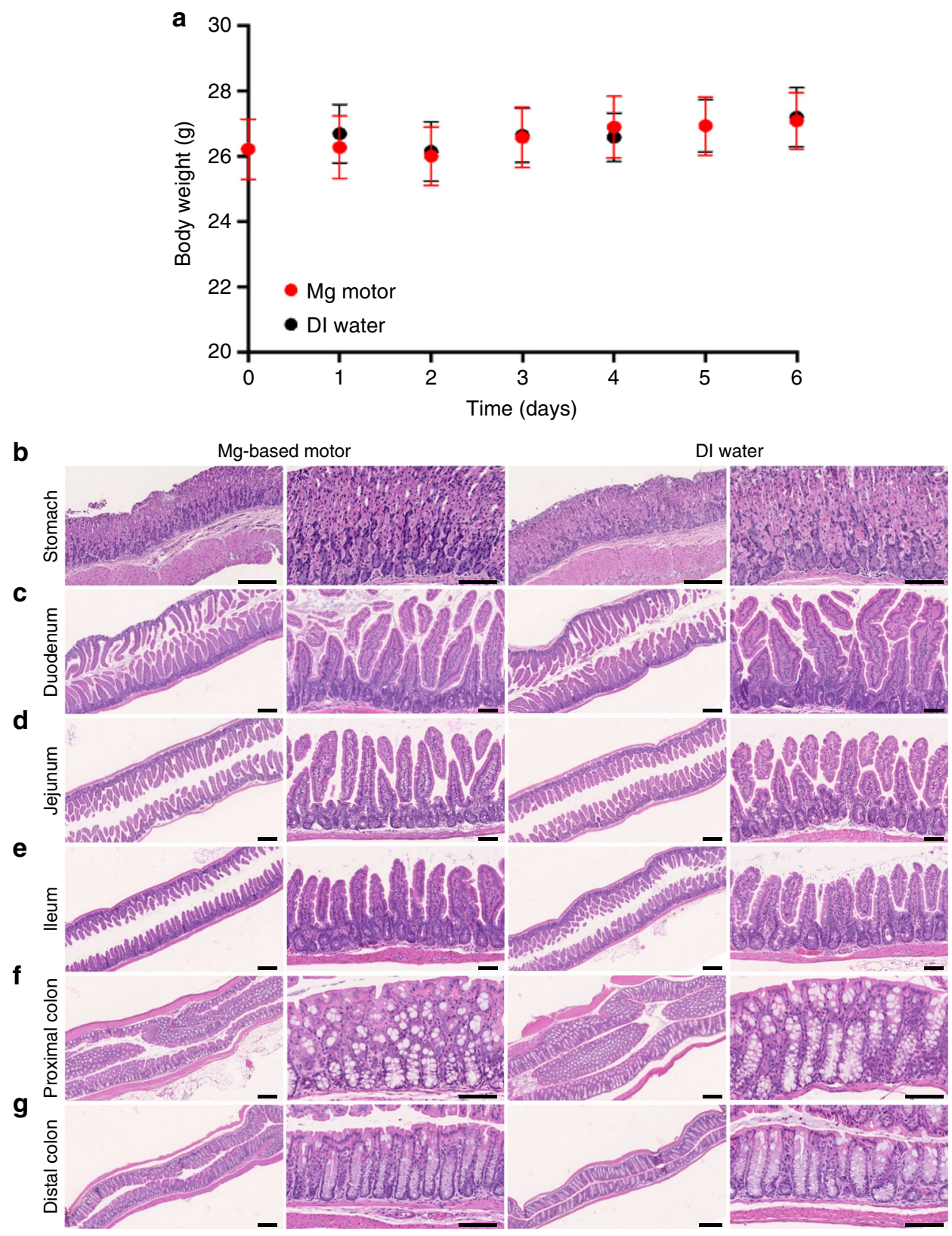

Fig. 5 In vivo toxicity evaluation of the Mg-based micromotors. Uninfected mice were orally administered with the Mg-based micromotors or DI water once daily for five consecutive days. a Mouse body weight log from day 0 to day 6 of the toxicity study. Error bars represent the s.d. of the mean ( $n=6$ ). On day 6, mice were killed and sections of the mouse stomach $\mathbf{b}$, small $\mathbf{c}-\mathbf{e}$ and large $\mathbf{f}, \mathbf{g}$ intestine tissues were processed for histological staining with hematoxylin and eosin (H\&E). Scale bars Mg-motor, 250 and $100 \mu \mathrm{m}$ (left and right column, respectively); DI water, 250 and $100 \mu \mathrm{m}$ (left and right column, respectively)

results indicate that micromotors may be adapted to the development of new and safe therapeutic treatments against stomach diseases such as $H$. pylori infection. As our early studies have shown that the Mg-based micromotors can propel efficiently and position precisely in the GI tract ${ }^{23}$, 24, we believe the presented motor-enabled delivery approach is promising to treat diverse GI tract diseases. Extending the propulsion methods with new alternative biocompatible fuels ${ }^{43}, 44$ or fuel-free actuation $^{11-13}$ might be able to expand the active-delivery concept to different parts of the body. We also envision that the micromotor approach will be useful for eliminating hard-to-treat bacterial biofilms, ${ }^{45}, 46$ with the efficient motor propulsion leading to biofilm penetration towards enhanced antibiotic delivery. Although the present results are promising, this work is still at its early stage. As a new active gastric delivery technology, future studies are required to further elucidate the micromotor's in vivo delivery performance and functions, and to compare with other standard therapies against $H$. pylori infection or other gastric diseases. Nonetheless, this work opens the door to the use of synthetic motors as an active-delivery platform for in vivo treatment of diseases and will likely trigger intensive research interests in this area.

\section{Methods}

Synthesis of Mg-based micromotors. The Mg-based micromotors were prepared using magnesium (Mg) microparticles (catalog \#FMW20, TangShan WeiHao Magnesium Powder Co.; average size, $20 \pm 5 \mu \mathrm{m}$ ) as the core. The $\mathrm{Mg}$ microparticles were initially washed with acetone to eliminate the presence of impurities. After being dried under a $\mathrm{N}_{2}$ current, the $\mathrm{Mg}$ microparticles were 
dispersed onto glass slides ( $2 \mathrm{mg}$ of $\mathrm{Mg}$ microparticles per glass slide), followed by ALD of $\mathrm{TiO}_{2}$ (at $100^{\circ} \mathrm{C}$ for 120 cycles) using a Beneq TFS 200 system. As such an ALD process utilizes gas phase reactants, it leads to uniform coatings over the $\mathrm{Mg}$ microparticles, whereas still leaving a small opening at the contact point of the particle to the glass slide. After that, the Janus micromotors were coated with 120 $\mu \mathrm{L}$ of $1 \%(\mathrm{w} / \mathrm{v})$ PLGA (Sigma-Aldrich, P2191) prepared in ethyl acetate (Sigma-Aldrich, 270989) and containing $40 \mathrm{mg} \mathrm{mL}^{-1}$ CLR (TCI CO., Ltd. C220). It should be noted that different CLR amounts (between $4 \mathrm{mg}$ and $6 \mathrm{mg}$ ) were tested to optimize the drug-loading. The PLGA@CLR coating was dried fast to avoid crystallization of the drug. Finally, the Janus micromotors were coated with a thin layer of $0.05 \%(\mathrm{w} / \mathrm{v})$ Chit (Sigma-Aldrich, C3646) prepared in water and containing $0.1 \%(\mathrm{w} / \mathrm{v})$ sodium dodecyl sulfate (SDS) (Sigma-Aldrich, 62862) and $0.02 \%(\mathrm{v} / \mathrm{v})$ acetic acid (Sigma-Aldrich, 695092), forming the outermost layer coated on the Mg microparticles. Finally, the Mg-based micromotors were collected by lightly scratching the microparticles off the glass slide.

\section{Synthesis of dye-loaded $\mathbf{M g}$-based micromotors. For performing the} characterization of the Mg-based micromotors along with the in vivo retention studies, fluorescent Mg-based micromotors were prepared by combining both 1\% PLGA and $0.05 \%$ Chit solutions with $5 \mu \mathrm{g} \mathrm{mL} \mathrm{m}^{-1} 1,1^{\prime}$-dioctadecyl-3,3,3',3'-tetramethylindodicarbocyanine, 4-chlorobenzenesulfonate salt (DiD, $\lambda_{\mathrm{ex}}=644 \mathrm{~nm} / \lambda_{\mathrm{en}}$ $=665 \mathrm{~nm}$, Life Technologies, D7757) and $1 \mu \mathrm{g} \mathrm{mL} \mathrm{L}^{-1}$ fluorescein isothiocyanatedextran (FITC, $\lambda_{\mathrm{ex}}=492 \mathrm{~nm} / \lambda_{\mathrm{em}}=520 \mathrm{~nm}$, Sigma-Aldrich, 46945) dyes, respectively. To compare with the $\mathrm{Mg}$-based micromotors, inert silica ( $\mathrm{Si}$ ) microparticles (Nanocs, Inc., Cat. No. Si01-20u-1; $20 \mu \mathrm{m}$ size) were used as core particles, following the same protocol described above.

Micromotor characterization. Bright-field and fluorescent images of the $\mathrm{Mg}$-based micromotors and inert silica microparticles (Supplementary Fig. 2) were captured using a EVOS FL microscope coupled with a $\times 20$ and $\times 40$ microscope objectives and fluorescence filters for red and green light excitation.

Scanning electron microscopy (SEM) images of the Mg-based micromotors were obtained with a Phillips XL30 ESEM instrument, using an acceleration voltage of $10 \mathrm{kV}$. EDX mapping analysis was performed using an Oxford EDX detector attached to SEM instrument and operated by INCA software.

Micromotor propulsion studies. Autonomous Mg-based micromotors propulsion in simulated gastric fluid (Sigma-Aldrich, 01651) was obtained by diluting 25 times the simulated gastric fluid according to the commercial specifications (final $\mathrm{pH}$ $\sim 1.3$ ), and adding $1 \%$ Triton X-100 (Fisher Scientific, FairLawn, NJ, USA) as surfactant. An inverted optical microscope (Nikon Eclipse 80i upright microscope) coupled with different microscope objectives $(\times 10, \times 20$, and $\times 40)$ and a QuantEM:512SC camera were used for recording the autonomous micromotor propulsion in the gastric fluid simulant. The speed of the Mg-based micromotors was characterized using the MetaMorph 7.1 software (Molecular Devices, Sunnyvale, CA, USA).

In vitro anti-H. pylori activity. H. pylori Sydney strain 1 (HPSS1) was cultured from frozen stock and routinely maintained on Columbia agar supplemented with $5 \%(\mathrm{vol} / \mathrm{vol})$ laked horse blood at $37^{\circ} \mathrm{C}$ under microaerobic conditions $\left(10 \% \mathrm{CO}_{2}\right.$, $85 \% \mathrm{~N}_{2}$, and $5 \% \mathrm{O}_{2}$ ). For experiments, broth cultures of $H$. pylori were prepared by subculturing fresh colonies from agar plates into brain-heart infusion (BHI) supplemented with $5 \%$ fetal bovine serum (FBS) and incubated overnight at $37^{\circ} \mathrm{C}$ under microaerobic conditions with moderate reciprocal shaking. An overnight broth culture of $\mathrm{H}$. pylori was centrifuged at $5000 \times \mathrm{g}$ for $10 \mathrm{~min}$ to obtain a bacterial pellet. After removal of culture medium by centrifugation, the obtained bacteria pellet was then suspended in an appropriate amount of fresh BHI with 5\% FBS for future use.

The bactericidal activity against $H$. pylori of free CLR and CLR-loaded Mg-based micromotors (PLGA@CLR-TiO $-\mathrm{Mg}$ ) were tested in vitro. All samples were treated in $0.1 \mathrm{~N} \mathrm{HCl}$ for $1 \mathrm{~h}$ and serially diluted to desired concentrations with $\mathrm{PBS}\left(\mathrm{pH}\right.$ 7). Bare Mg-based micromotors ( $\mathrm{PLGA}-\mathrm{TiO}_{2}-\mathrm{Mg}$ ) with corresponding amount of micromotors were used as negative control.

The samples were added with $1 \times 10^{6} \mathrm{CFU} \mathrm{mL}^{-1} \mathrm{H}$. pylori in BHI with $5 \% \mathrm{FBS}$ to make final concentrations of $0-16 \mu \mathrm{g} \mathrm{mL}^{-1} \mathrm{CLR}$, followed by incubation at $37^{\circ} \mathrm{C}$ under microaerobic conditions with moderate reciprocal shaking for $24 \mathrm{~h}$. Then, a series of 10 -fold dilutions of the bacterial suspension was prepared, and inoculated onto a Columbia agar plates supplemented with $5 \%$ laked horse blood. The plates were cultured for 4 days before the colony-forming unit (CFU) of H. pylori was quantified. All measurements were made in triplicate.

In vivo micromotor retention. Prior to the experiment, C57BL/6 mice $(n=3)$ were fed with alfalfa-free food from LabDiet (St Louis, MO, USA) for 2 weeks. The in vivo retention study was performed by using dye-loaded $\mathrm{Mg}$-based micromotors prepared by the protocol described above. A $0.3 \mathrm{~mL}$ suspension of $\mathrm{Mg}$-based micromotors with DiD-labeled PLGA and FITC-labeled chitosan coatings were intragastrically administered. A group of mice was administered with DI water as a negative control. Following $30 \mathrm{~min}$ and $2 \mathrm{~h}$ of oral administrations, the mice were killed and their entire stomachs were excised and cut opened along the greater curvature. Then, the tissues were rinsed with PBS, flattened, and visualized using a Keyence BZ-X700 fluorescence microscope. The bright-field and corresponding fluorescence images were obtained at 665 and $520 \mathrm{~nm}$ (DiD and FITC, respectively) for each sample. Subsequently, the tissues were transferred to $1 \mathrm{~mL}$ PBS and homogenized. Analysis of the amount of micromotors retained in the stomachs was carried out by measuring the fluorescence intensity of their embedded DiD-labeled PLGA and FITC-labeled chitosan using Synergy Mx fluorescent spectrophotometer (Biotek, Winooski, VT, USA). All animal experiments were in compliance with the University of California San Diego Institutional Animal Care and Use Committee (IACUC) regulations.

In vivo therapeutic efficacy against $\mathbf{H}$. pylori infection. Six-week-old C57BL/6 male mice were purchased from the Jackson Laboratory (Bar Harbor, ME, USA) Each C57BL/6 mouse received $0.3 \mathrm{~mL}$ of $1 \times 10^{9} \mathrm{CFU} \mathrm{mL}^{-1} \mathrm{H}$. pylori in BHI broth administered intragastrically through oral gavage every $48 \mathrm{~h}$, repeated three times (on day 3,5, and 7, respectively), and the infection was allowed to develop for 2 weeks $^{39}$. For the in vivo anti-H. pylori therapeutic study, mice were randomly divided in five treatment groups $(n=6)$ to be orally administered once daily for five consecutive days with CLR-loaded Mg-based micromotors, CLR-loaded inert silica microparticles, free CLR+PPI, blank Mg-based micromotors or DI water. For free CLR+PPI group, each day of treatment mice were first administered with omeprazole (a PPI) through oral gavage at a dose of $400 \mu \mathrm{mol} \mathrm{kg}^{-139,40-42}$, followed by a lag time of $30 \mathrm{~min}$ before administration of CLR. CLR-loaded Mg-based micromotors, CLR-loaded inert silica microparticles and free CLR (with $30 \mathrm{mg} \mathrm{kg}^{-1}$ CLR dosage) were also administered through oral gavage once daily for five consecutive days ${ }^{39}$. Blank Mg-based micromotors and DI water served as movement control and negative control, respectively. Forty-eight hours after last administration ${ }^{39,47-49}$, mice were killed and stomachs were excised from the abdominal cavity. The stomachs were cut along the greater curvature, and the gastric content were removed and rinsed with PBS. For H. pylori recovery, each gastric tissue was weighed before suspended in $200 \mu \mathrm{L}$ PBS and homogenized. The homogenate was serially diluted and spotted onto Columbia agar plate with $5 \%$ laked horse blood and Skirrow's supplement $\left(10 \mu \mathrm{g} \mathrm{mL}^{-1}\right.$ vancomycin, $5 \mu \mathrm{g} \mathrm{mL} \mathrm{L}^{-1}$ trimethoprim lactate, and $2500 \mathrm{IU} / \mathrm{L}$ polymyxin B; Oxiod). The plates were then incubated at $37^{\circ} \mathrm{C}$ under microaerobic conditions for 5 days, and bacterial colonies were enumerated. Statistical analysis was performed using one-way ANOVA. No statistical methods were used to predetermine sample size. Studies were done in a non-blinded fashion. Replicates represent different mice subjected to the same treatment $(n=6)$. All animal experiments were in compliance with the University of California San Diego Institutional Animal Care and Use Committee (IACUC) regulations.

Toxicity evaluation of $\mathbf{M g}$-based micromotors. To evaluate the acute toxicity of the Mg-based micromotors in vivo, uninfected C57BL/6 male mice $(n=6)$ weighing $25-30 \mathrm{~g}$ were orally administered with CLR-loaded micromotors once daily for five consecutive days. Mice administered with DI water were tested in parallel as a negative control. During the experimental period, the mouse body weight was monitored by weighing the mice daily. On day 6 , mice were killed and sections of the mouse stomach, small and large intestine tissues were processed for histological examination. The stomach was cut open along the greater curvature, and the gastric content was removed. The small and large intestines were cut to small sections as duodenum, jejunum, ileum, proximal, and distal colon and rinsed inside with PBS to remove internal residues. The longitudinal tissue sections were fixed in neutral-buffered $10 \%$ (vol/vol) formalin for $15 \mathrm{~h}$, transferred into $70 \%$ ethanol, and embedded in paraffin. The tissue sections were cut with $5 \mu \mathrm{m}$ thickness and stained with $\mathrm{H} \& \mathrm{E}$ assay. The stained sections were visualized by Hamamatsu NanoZoomer 2.0HT and the images processed using NDP viewing software. All animal experiments were in compliance with the University of California San Diego Institutional Animal Care and Use Committee (IACUC) regulations.

Data availability. The authors declare that all relevant data supporting the findings of this study are available within the article and its supplementary information files as well from the authors upon reasonable request.

Received: 20 February 2017 Accepted: 19 June 2017

Published online: 16 August 2017

\section{References}

1. Wang, J. Nanomachines: fundamentals and Applications (Wiley, 2013).

2. Guix, M., Mayorga-Martinez, C. C. \& Merkoçi, A. Nano/micromotors in (bio) chemical science applications. Chem. Rev. 114, 6285-6322 (2014).

3. Mei, Y., Solovev, A. A., Sanchez, S. \& Schmidt, O. G. Rolled-up nanotech on polymers: from basic perception to self-propelled catalytic microengines. Chem Soc. Rev. 40, 2109-2119 (2011).

4. Sánchez, S., Soler, L. \& Katuri, J. Chemically powered micro- and nanomotors. Angew. Chem. Int. Ed. 54, 1414-1444 (2015). 
5. Nelson, B. J., Kaliakatsos, I. K. \& Abbott, J. J. Microrobots for minimally invasive medicine. Annu. Rev. Biomed. Eng. 12, 55-85 (2010).

6. Li, J., Rozen, I. \& Wang, J. Rocket science at the nanoscale. ACS Nano 10, 5619-5634 (2016).

7. Abdelmohsen, L. K. E. A., Peng, F., Tu, Y. \& Wilson, D. A. Micro- and nano-motors for biomedical applications. J. Mater. Chem. B 2, 2395-2408 (2014).

8. Sundararajan, S., Lammert, P. E., Zudans, A. W., Crespi, V. H. \& Sen, A. Catalytic motors for transport of colloidal cargo. Nano. Lett. 8, 1271-1276 (2008).

9. $\mathrm{Wu}, \mathrm{Z}$. et al. Self-propelled polymer-based multilayer nanorockets for transportation and drug release. Angew. Chem. Int. Ed. 52, 7000-7003 (2013).

10. Gao, W. \& Wang, J. Synthetic micro/nanomotors in drug delivery. Nanoscale 6 , 10486-10494 (2014).

11. Servant, A., Qiu, F., Mazza, M., Kostarelos, K. \& Nelson, B. J. Controlled in vivo swimming of a swarm of bacteria-like microrobotic flagella. Adv. Mater. 27, 2981-2988 (2015).

12. Felfoul, O. et al. Magneto-aerotactic bacteria deliver drug-containing nanoliposomes to tumour hypoxic regions. Nat. Nanotechnol. 11, 941-947 (2016).

13. Esteban-Fernández de Ávila, B. et al. Acoustically propelled nanomotors for intracellular siRNA delivery. ACS Nano 10, 4997-5005 (2016).

14. Kagan, D. et al. Acoustic droplet vaporization and propulsion of perfluorocarbon-loaded microbullets for targeted tissue penetration and deformation. Angew. Chem. Int. Ed. 51, 7519-7522 (2012).

15. Leong, T. G. et al. Tetherless thermobiochemically actuated microgrippers. Proc. Natl Acad. Sci. USA 106, 703-708 (2009).

16. Balasubramanian, S. et al. Micromachine-enabled capture and isolation of cancer cells in complex media. Angew. Chem. Int. Ed. 50, 4161-4164 (2011).

17. Mallouk, T. E. \& Sen, A. Powering nanorobots. Sci. Am. 300, 72-77 (2009).

18. Esteban-Fernández de Ávila, B. et al. Single cell real-time miRNAs sensing based on nanomotors. ACS Nano 9, 6756-6764 (2015).

19. Srivastava, S. K., Guix, M. \& Schmidt, O. G. Wastewater mediated activation of micromotors for efficient water cleaning. Nano. Lett. 16, 817-821 (2016).

20. Hoop, M. et al. Magnetically driven silver-coated nanocoils for efficient bacterial contact killing. Adv. Funct. Mater. 26, 1063-1069 (2016).

21. Soler, L., Magdanz, V., Fomin, V. M., Sanchez, S. \& Schmidt, O. G. Self-propelled micromotors for cleaning polluted water. ACS Nano 7, 9611-9620 (2013).

22. Gao, W. et al. Artificial micromotors in the mouse's stomach: a step toward in vivo use of synthetic motors. ACS Nano 9, 117-123 (2015).

23. Li, J. et al. Enteric micromotor can selectively position and spontaneously propel in the gastrointestinal tract. ACS Nano 10, 9536-9542 (2016).

24. $\mathrm{Li}$, J. et al. Micromotors spontaneously neutralize gastric acid for $\mathrm{pH}$-responsive payload release. Angew. Chem. 129, 2188-2193 (2017).

25. Walker, D., Käsdorf, B. T., Jeong, H.-H., Lieleg, O. \& Fischer, P. Enzymatically active biomimetic micropropellers for the penetration of mucin gels. Sci. Adv. 1, e1500501 (2015)

26. Salama, N. R., Hartung, M. L. \& Müller, A. Life in the human stomach: persistence strategies of the bacterial pathogen Helicobacter pylori. Nat. Rev. Microbiol. 11, 385-399 (2013).

27. Sachs, G., Weeks, D. L., Melchers, K. \& Scott, D. R. The gastric biology of Helicobacter pylori. Annu. Rev. Physiol. 65, 349-369 (2003).

28. Holtmann, G., Cain, C. \& Malfertheiner, P. Gastric Helicobacter pylori infection accelerates healing of reflux esophagitis during treatment with the proton pump inhibitor pantoprazole. Gastroenterology. 117, 11-16 (1999).

29. Olbe, L., Carlsson, E. \& Lindberg, P. A proton-pump inhibitor expedition: the case histories of omeprazole and esomeprazole. Nat. Rev. Drug. Discov. 2, 132-139 (2003)

30. Richardson, P., Hawkey, C. J. \& Stack, W. A. Proton pump inhibitors. Pharmacology and rationale for use in gastrointestinal disorders. Drugs 56, 307-335 (1998)

31. Moayyedi, P. \& Leontiadis, G. I. The risks of PPI therapy. Nat. Rev. Gastroenterol. Hepatol. 9, 132-139 (2012).

32. Ho, P. M. et al. Risk of adverse outcomes associated with concomitant use of clopidogrel and proton pump inhibitors following acute coronary syndrome. IAMA 301, 937 (2009).

33. Sheen, E. \& Triadafilopoulos, G. Adverse effects of long-term proton pump inhibitor therapy. Dig. Dis. Sci. 56, 931-950 (2011).

34. Yang, Y.-X., Lewis, J. D., Epstein, S. \& Metz, D. C. Long-term proton pump inhibitor therapy and risk of hip fracture. JAMA 296, 2947 (2006).

35. Martirosyan, A., Olesen, M. J. \& Howard, K. A. Chitosan-based nanoparticles for mucosal delivery of RNAi therapeutics. Adv. Genet. 88, 325-352 (2014).

36. $\mathrm{Li}$, J. et al. Water-driven micromotors for rapid photocatalytic degradation of biological and chemical warfare agents. ACS Nano 8, 11118-11125 (2014).

37. Watanabe, K. et al. Effect of sucralfate on antibiotic therapy for Helicobacter pylori infection in mice. Antimicrob. Agents Chemother. 48, 4582-4588 (2004).
38. Obonyo, M., Guiney, D. G., Harwood, J., Fierer, J. \& Cole, S. P. Role of gamma interferon in Helicobacter pylori induction of inflammatory mediators during murine infection. Infect. Immun. 70, 3295-3299 (2002).

39. Thamphiwatana, S., Gao, W., Obonyo, M. \& Zhang, L. In vivo treatment of Helicobacter pylori infection with liposomal linolenic acid reduces colonization and ameliorates inflammation. Proc. Natl Acad. Sci. USA 111, 17600-17605 (2014).

40. Lameris, A. L. L., Hess, M. W., van Kruijsbergen, I., Hoenderop, J. G. J. \& Bindels, R. J. M. Omeprazole enhances the colonic expression of the $\mathrm{Mg}^{2+}$ transporter TRPM6. Pflugers Arch-Eur. J Physiol 465, 1613-1620 (2013).

41. Correia, M. et al. Docosahexaenoic acid inhibits Helicobacter pylori growth in vitro and mice gastric mucosa colonization. PLoS ONE 7, e35072 (2012).

42. Tran, C. D. et al. Novel combination therapy for the eradication of Helicobacter pylori infection in a mouse model. Scand. J. Gastroenterol. 45, 1424-1430 (2010).

43. Mou, F. et al. Autonomous motion and temperature controlled drug delivery of $\mathrm{Mg} / \mathrm{Pt}$-poly(N-isopropylacrylamide) Janus micromotors driven by simulated body fluid and blood plasma. ACS Appl. Mater. Interfaces 6, 9897-9903 (2014).

44. Dey, K. K. et al. Micromotors powered by enzyme catalysis. Nano Lett. 15, 8311-8315 (2015).

45. Subbiahdoss, G. et al. Magnetic targeting of surface-modified superparamagnetic iron oxide nanoparticles yields antibacterial efficacy against biofilms of gentamicin-resistant staphylococci. Acta Biomater. 8, 2047-2055 (2012).

46. Davies, D. Understanding biofilm resistance to antibacterial agents. Nat. Rev. Drug Discov. 2, 114-122 (2003).

47. Ping, Y. et al. Engineering bioinspired bacteria-adhesive clay nanoparticles with a membrane-disruptive property for the treatment of Helicobacter pylori infection. Nanoscale 8, 16486-16498 (2016).

48. Lin, Y.-H., Feng, C.-L., Lai, C.-H., Lin, J.-H. \& Chen, H.-Y. Preparation of epigallocatechin gallate-loaded nanoparticles and characterization of their inhibitory effects on Helicobacter pylori growth in vitro and in vivo. Sci. Technol. Adv. Mater. 15, 045006 (2014).

49. Lin, Y.-H. et al. Genipin-cross-linked fucose-chitosan/heparin nanoparticles for the eradication of Helicobacter pylori. Biomaterials 34, 4466-4479 (2013).

\section{Acknowledgements}

This work is supported by the Defense Threat Reduction Agency Joint Science and Technology Office for Chemical and Biological Defense (Grant Numbers HDTRA1-13-10002 and HDTRA1-14-1-0064) and by the National Institute of Diabetes and Digestive and Kidney Diseases of the National Institutes of Health (Award Number R01DK095168)

\section{Author contributions}

J.W., L.Z., B.E.-F.d.A., P.A., J.L., and S.T. conceived the idea and designed the experiments. B.E.-F.d.A., P.A., J.L., M.A.L.-R., D.E.R.-H., S.T., C.C., J.D., R.S., and V.R. performed all the experiments. The manuscript was written by B.E.-F.d.A., P.A., J.L., J.W., and L.Z. All authors contributed to the general discussion and reviewed the manuscript. M.O. provided the H. pylori SS1 strain for this work and participated in the design of $\mathrm{H}$ pylori infection studies.

\section{Additional information}

Supplementary Information accompanies this paper at doi:10.1038/s41467-017-00309-w.

Competing interests: The authors declare no competing financial interests.

Reprints and permission information is available online at http://npg.nature.com/ reprintsandpermissions/

Change history: A correction to this article has been published and is linked from the HTML version of this paper.

Publisher's note: Springer Nature remains neutral with regard to jurisdictional claims in published maps and institutional affiliations.

cc) (i) Open Access This article is licensed under a Creative Commons BY Attribution 4.0 International License, which permits use, sharing adaptation, distribution and reproduction in any medium or format, as long as you give appropriate credit to the original author(s) and the source, provide a link to the Creative Commons license, and indicate if changes were made. The images or other third party material in this article are included in the article's Creative Commons license, unless indicated otherwise in a credit line to the material. If material is not included in the article's Creative Commons license and your intended use is not permitted by statutory regulation or exceeds the permitted use, you will need to obtain permission directly from the copyright holder. To view a copy of this license, visit http://creativecommons.org/ licenses/by/4.0/.

(C) The Author(s) 2017 